In diesem Artikel wird auf jene Punkte der neuen Spitalfinanzierung eingegangen, die weiterhin offen sind. Zwar decken sie sich mit den Anliegen der FMH, doch werden sie auf eine unterschiedliche Art und Weise angegangen. Wie die Autoren festhalten, bringen uns reine Absichtserklärungen nicht weiter. Einzig mit einer objektiven, emotionslosen Analyse der Situation lässt sich eine Haltung entwickeln, mit der es möglich sein wird, Einfluss auf unsere Partner zu nehmen und so zu Resultaten zu gelangen, da sich die Politik zurzeit kaum für das Thema interessiert.

Ausgehend von diesem Grundsatz, setzt sich die FMH sehr aktiv, aber ohne Schwarzmalerei dafür ein, dass die Veränderungen ab dem 1.1.2012 unter Bedingungen umgesetzt werden, mit denen unsere therapeutische Freiheit gewahrt bleibt und die sich für die Patientinnen und Patienten nicht nachteilig auswirken. Für die Forderungen, die am Ende dieses Artikels aufgestellt werden, liegen bereits Lösungen vor oder werden gegenwärtig entwickelt. Auch wenn sie zuweilen von unseren Erwartungen abweichen, sind sie nicht grundsätzlich negativ. Einzig die Idee einer vereinfachten SwissDRG-Struktur macht keinen Sinn: Die Version 1.0 liegt bereit, um in allen Spitälern eingeführt zu werden, und wird nicht bloss für ausgewählte Fälle zur Anwendung gelangen.

Dr. med. Pierre-François Cuénoud Mitglied des FMH-Zentralvorstands Verantwortlicher Ressort SwissDRC

\title{
SwissDRG: Übergangsregelung - Ende einer Hoffnung?
}

Christian Hess, Reto Krapf, Danielle Lemann, Urs Strebel

Für die Petitionsgruppe (alphabetisch)
Während der Vorstand der FMH die Einführung der SwissDRG grundsätzlich unterstützte und die FMH beträchtliche Ressourcen in das Projekt investierte, hat sich Mitte 2008 eine Gruppe aus Ärzten, Psychotherapeuten, Physiotherapeuten und anderen verwandten Berufsgruppen zusammen mit Patientenvertretern, Politikern und Gewerkschaftsvertretern gebildet, die ein Moratorium der DRG-Einführung forderte, bis folgende Fragen und Probleme geklärt und gelöst sind:

Versorgungssicherheit, Datenschutz, Arbeitsbedingungen für das Gesundheitspersonal, Investitionskosten, Begleitforschung, Finanzierung der ärztlichen Weiterbildung, Auswirkungen auf die vor- und nachgelagerten Bereiche wie Rehabilitation, Langzeitpflege, Hausärzte und Spitex. Des Weiteren wurde die
«Ökonomisierung der Medizin» lanciert. Die NEK-CNE hatte ihre Hauptbedenken bereits am 3.9.2008 in einem Artikel in der Schweizerischen Ärztezeitung [1] veröffentlicht.

Da das Echo auf beide Initiativen vor allem auch in politischen Kreisen gering blieb und es nicht gelang, eine breite öffentliche Diskussion zu lancieren, hat die Moratoriumsgruppe Anfang 2011 versucht, (a) mittels einer Petition der Ärzteschaft an den Zentralvorstand der FMH die Meinung der FMH-Basis in Erfahrung zu bringen [2] (es gingen rund 3800 Unterschriften von FMH-Mitgliedern ein), und (b) in der Junisession politisch Einfluss zu nehmen, nachdem ein Hearing bei der SGK-N ebenfalls ohne sichtbares Resultat verlief. Ziel war eine parlamentarische Motion, welche die Einführung der SwissDRG in zwei

\section{«Blosse Erklärungen sind unseres Erachtens zu wenig und führen zur zunehmenden Unglaubwürdigkeit der FMH in dieser Sache.»}

Verlagerung von Stellen vom Krankenbett in die Verwaltung und die generelle Veradministrierung moniert. Eine Online-Plattform warb für Unterschriften von Personen, die diese Anliegen ebenfalls unterstützen, und konnte rund 5000 Unterschriften sammeln (www.drg-moratorium.ch).

Im Anschluss an ein Hearing der Nationalen Ethikkommission im Bereich der Humanmedizin NEK-CNE vom 18.9.2008, zu dem Initianten des DRG-Moratoriums ebenfalls eingeladen waren, wurde durch die NEK-CNE ein Symposium zum Thema
Schritten forderte: Beginn mit einer begrenzten Zahl gutdefinierter Diagnosen und Prozeduren (im Wesentlichen häufige Wahleingriffe), die mit schweizerischen Finanzzahlen verlässlich hinterlegt sind, und während dieser Lernphase eine Begleitforschung durchzuführen, bevor der Schritt, zu einem späteren Zeitpunkt, in ein flächendeckendes System vollzogen würde (Nationalrätin Christine Goll und Nationalrätin Yvonne Gilli haben in der Junisession zwei entsprechende Motionen eingereicht. Die erste wurde von Bundesrat und Parlament abgelehnt; die zweite 
wird vom Parlament noch behandelt, nachdem sie der Bundesrat ebenfalls abgelehnt hat.

Parallel zu diesen Bemühungen machte sich der VSAO an der Ärztekammer der FMH vom 26. Mai 2011 für das Moratorium stark, das zugunsten einer Erklärung der FMH knapp abgelehnt wurde. Diese Erklärung ist in der SÄZ 25/2011 unter dem Titel «Patientenversorgung ist nur mit Übergangsregelung gesichert» [3] publiziert worden. Darin heisst es, dass folgende Fragen vor einer definitiven Einführung gelöst sein müssen: Zusatzentgelte, Investitionskosten, ärztliche Weiterbildung, Datenschutz und Begleitforschung. Zudem wird der Einbezug der Ärzte in die Spital- und Versorgungsplanung gefordert.

Wir von der DRG-Moratoriums/Petitions-Gruppe mussten im August 2011 zur Kenntnis nehmen, dass die Einführung der DRGs per 1.1.2012 nicht mehr zu verhindern ist.

Da sich aber fast alle unsere Forderungen mit den Forderungen der FMH deckten und nach wie vor ungelöst sind, suchten wir das Gespräch mit dem Zentralvorstand der FMH und boten unsere Unterstüt- ökonomisierende Ansatz ist zudem gefährlich, weil er vorgibt, mit wenigen numerischen Daten gerecht abzugelten und steuern zu können. Die grossen Versprechungen der Transparenz, der Kosteneinsparung und der verbesserten Qualität sind schon längst als politische Slogans demaskiert und werden auch von den Befürwortern kaum mehr bemüht.

Wir fordern die FMH auf, endlich aktiv zu werden und zu retten, was eben noch zu retten ist. Folgende Minimalforderungen müssen gegenüber der Politik gestellt werden:

- Lösung des Datenschutzproblems durch Schaffung einer Institution unabhängiger Vertrauensärzte.

- Am 1. Januar 2012 wird mit einer «DRG-light»Version begonnen (Beschränkung auf die häufigen Wahleingriffe).

- Die Begleitforschung untersucht in diesem Modell den konkreten Effekt auf Kosten und die Frage der Mengenausweitung.

- Nach Vorliegen ihrer Resultate und Auswertung der Erfahrungen wird nach 3 Jahren über eine all-

\section{«Wir fordern die FMH auf, endlich aktiv zu werden und zu retten, was eben noch zu retten ist.»}

zung beim Ausformulieren und Konkretisieren der Übergangsregelungen an.

Am 13.10.2011 trafen sich Jacques de Haller, Pierre-François Cuénoud, Christian Hess, Reto Krapf, Danielle Lemann und Urs Strebel in Bern, um die gemeinsamen Punkte zu klären. Es herrschte Übereinstimmung, dass die erwähnten Punkte nach wie vor nicht befriedigend gelöst sind und es bis zum 1.1.2012 auch nicht sein werden. Nicht einigen konnten wir uns allerdings darüber, welche konkreten Schritte und Massnahmen der Vorstand der FMH nun ergreifen soll. Blosse Erklärungen sind unseres Erachtens zu wenig und führen zur zunehmenden Unglaubwürdigkeit der FMH in dieser Sache.

Zusammengefasst sind wir der dezidierten Meinung, dass der Ernst der Lage von (zu)vielen - inklusive der FMH-Spitze - immer noch nicht erkannt wird. Es ist evident, dass die Einführung der DRG in vorgesehener/em Form und Umfang zum Schaden der Patienten, des Personals, der ärztlichen Weiterbildung und damit des schweizerischen Gesundheitswesens gereicht, einzig, um einem betriebswirtschaftlichen Ansatz zum Durchbruch zu verhelfen. Der fällige Erweiterung von «DRG-light» entschieden. - Die Kosten der ärztlichen Weiterbildungen sind genau und realistisch erfasst und gesamtschweizerisch geregelt entgolten.

- Die Frage der Investitionskosten muss so geregelt sein, dass kein Investitionsstau entsteht und die Spitäler nicht gezwungen werden, Behandlungsgelder für Investitionen umzuleiten.

An dieser Stelle danken wir allen Petitions- und Moratoriumsunterzeichnenden für ihre Unterstützung.

\section{Literatur}

1 Brauer S. Zur Einführung von diagnosebezogenen Fallpauschalen in Schweizer Spitälern. Schweiz Ärztezeitung. 2008;89(38):1533-6.

2 Hess C, Krapf R, Strebel U. Für die Einführung der Fallpauschalen (DRG) am 1. Januar 2012 ist es zu früh! - Petition an den Zentralvorstand der FMH. Schweiz Ärztezeitung. 2011;92(11):423.

3 Erklärung der FMH zur Einführung der neuen Spitalfinanzierung am 1.1.2012. Patientenversorgung ist nur mit Übergangsregelung gesichert. Schweiz Ärztezeitung. 2011;92(25):924-5. 